\title{
THE CEM-UT RAPID-FIRE COMPULSATOR TEST RESULTS AND PERFORMANCE EVALUATION
}

\author{
By: \\ D. E. Perkins \\ S. B. Pratap \\ M. L. Spann \\ R. F. Thelen \\ M. D. Werst
}

Seventh IEEE Pulsed Power Conference, Monterey, CA, June 11-14, 1989.

IEEE Transactions on Magnetics, vol. 27, no. 1, January 1991, pp. 74-79

PN - 150

Center for Electromechanics

The University of Texas at Austin

PRC, Mail Code R7000

Austin, TX 78712

(512) 471-4496 


\author{
D. E. Perkins, S. B. Pratap, M. L. Spann, \\ R. F. Thelen, and M. D. Werst \\ Center for Electromechanics \\ The University of Texas at Austin \\ Austin, TX 78758-4497
}

Abstract: Since its inception in 1978, much attention has focused on compulsators as an alternative to capacitors and homopolar generators as a pulsed power supply. In addition to high voltage, high peak power, and large power densities, compulsators offer the advantage of supplying repetitive pulses without the need for external power conditioning. These repetitive power pulses are well suited for driving laser flashlamps, electromagnetic and electrothermal launchers, and particle beam accelerators.

In April of 1986, fabrication and installation of a 1 megajoule (MJ) per pulse, rapid-fire compulsator was completed at the Center for Electromechanics at The University of Texas at Austin (CEM-UT). A test program was initiated and, since then, a substantial volume of machine performance data has been collected. This paper presents the mechanical and electrical performance data of the compulsator. Included are measurements of rotor critical frequency and bearing performance. Also shown are open circuit voltage characteristics and current waveform characteristics during discharge into low impedance loads.

\section{Introduction}

In a program to develop a rapid fire electromagnetic launcher, engineers at CEM-UT have incorporated a ferromagnetic based compulsator rated at $2.0 \mathrm{kV}$ that is capable of delivering ten, $2.0 \mathrm{~ms}$ current pulses of 1.0 MA peak amplitude. The machine (fig. 1) occupies a volume of approximately $1.7 \mathrm{~m}^{3}$, weighs $11,000 \mathrm{~kg}$, and, at an operating speed of $3,600 \mathrm{rpm}$, has presently delivered $1.0 \mathrm{MJ}$ of energy to a low impedance load and generated in excess of $800 \mathrm{MW}$.

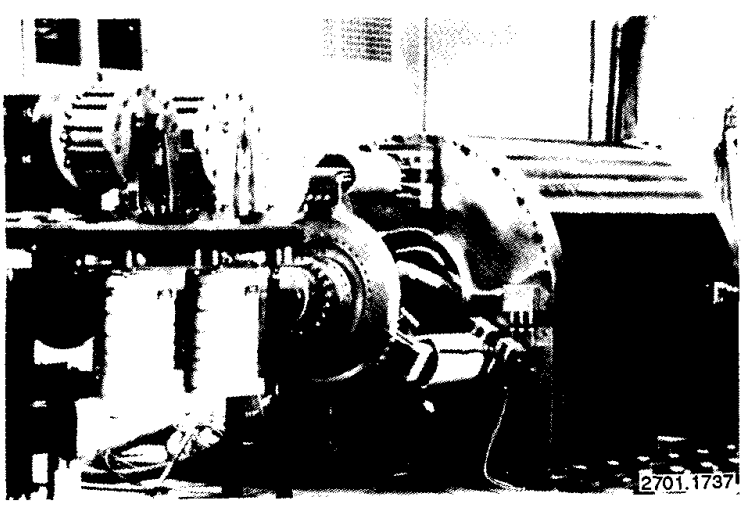

Figure 1. $1 \mathrm{MJ} /$ pulse, iron-core compulsator

The CEM-UT compulsator uses a six pole, rotating excitation field winding on a high strength, alloy steel rotor and a lap wound armature winding on a laminated steel stator. Passive compensation is achieved with the use of a conductive shield on the periphery of the rotor. Figure 2 shows a sectioned view of the machine.

The excitation winding is composed of 68 turns per pole of $0.64-x$ $0.64-\mathrm{cm}$ solid copper wire which is coated with an insulating film. The wire is also wrapped with fiberglass tape which is epoxy impregnated after rotor assembly is complete. This provides the winding with mechanical integrity under centrifugal loading. The excitation winding is pulse rated and may carry $1.2 \mathrm{kA}$ for $6 \mathrm{~s}$. The total on time of the field coil at rated field current is $10 \mathrm{~s}$ to allow the current to ramp up and to allow time for the eddy currents in the poles to decay

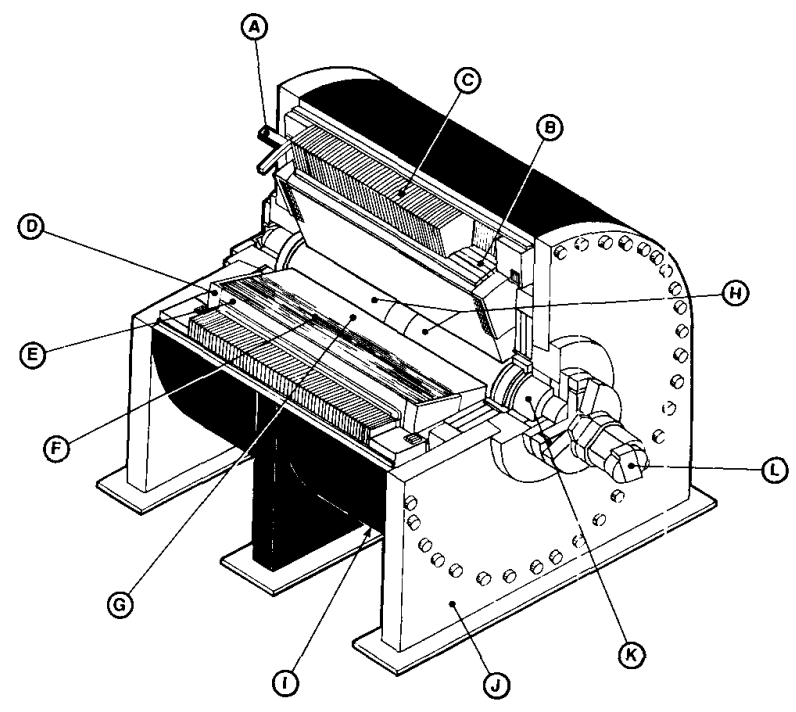

2701.1353

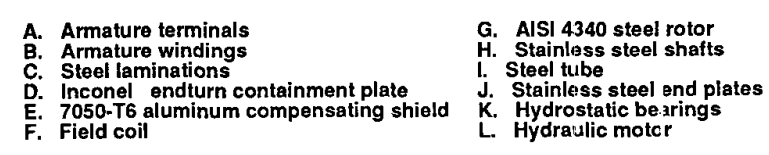

2701.1353.TXT

Figure 2. Sectional view of a passively compensated rotat.ng field machine

The armature winding is made of six lap wound coils which are connected in parallel. Each coil is made of $7 \times 7 \times 16 \times \# 24 \wedge W G$ copper litz wire which is fully transposed. The litz wire is wound with a half lap wrap of fiberglass tape and is epoxy impregnated. This provides the turn to turn and part of the ground plane insulation. The ground plane insulation is further enhanced with an epoxy impregnated fiberglass mat. The end turns of the armature winding are embedded in slots machined in G-10 rings on both ends of the compulsator

The shield, which provides passive compensation, is an essential part of the machine. The primary purpose of the shield is to provide a highly conductive surface, however, mechanical constraints limit material choices to those of less than optimum conductivity. The shield undergoes the following types of loading:

1) centrifugal loading due to rotation,

2) axial loading during discharge, 
3) compressive loading during discharge,

4) a hoop stress due to shrink fit on the rotor, and

5) radial growth due to heating by the flow of compensating and eddy currents.

Centrifugal loading can be minimized by using a low density material such as aluminum. In order to counteract the thermal and centrifugal radial growth and still transmit the discharge torque, the shield is shrunk onto the rotor with an interference fit which results in a significant hoop stress. Aluminum 7050 was selected as the shield material which compromises the conductivity to some extent, but satisfies all the mechanical criteria.

Due to the transient nature of the compulsator duty cycle, subcritical rotor dynamic behavior is desirable. The primary mode of vibration is radial and hydrostatic bearings have been selected in order to provide the requisite radial stiffness. The thrust bearings are also hydrostatic.[1]

Initial machine testing includes an analysis of the static and dynamic behavior of the hydrostatic bearings and the rotor. Bearing pocket pressures are recorded and rotor dynamic response measurements are given in the axial and radial modes to determine the machine critical frequency.

Following these mechanical tests, a series of open circuit electrical tests was performed with the rotor spinning at $500 \mathrm{rpm}$ Current was applied to the rotating field coil and open circuit voltage was measured. Several tests were performed at various levels of field current in order to produce an open circuit magnetic saturation curve for the machine. Since high machine current is a result of compensation, an analysis of the effectiveness of the compensating shield is included along with a discussion of the open circuit test results.

After completion of open circuit testing, the compulsator was connected to a low impedance load. A trigger circuit was designed to initiate a discharge at a precise phase angle of the open circuit voltage. A brief description of this circuit is given. Presently, the compulsator has been discharged at $90 \%$ full field excitation and $75 \%$ of full speed. Other tests include repetitive discharges at lower energies (50\% of full speed and $75 \%$ full excitation). Discharge data collected and to be discussed includes machine voltage, total current delivered, total energy produced, and peak power developed.

\section{Mechanical Performance}

Subcritical machine performance is desired to avoid continuously traversing through critical frequencies due to the pulsed operating duty. Since the compulsator uses hydrostatic bearings, it was a simple matter to check the frequency response of the rotor by applying an impulsive load and monitoring bearing pocket pressures and rotor displacement transducers.

For the axial (thrust) bearings, access to the rotor was obtained by removing a hydraulic motor from one end of the machine. A $1.4 \mathrm{~kg}$ ( $3 \mathrm{lb}$ ) hammer was used to strike the rotor shaft to excite the axial vibration mode and a damped response curve was obtained. With a pocket pressure of $3,688 \mathrm{kPa}(535 \mathrm{psi})$ and oil temperature of $21^{\circ} \mathrm{C}$ $\left(70^{\circ} \mathrm{F}\right)$, an axial frequency of $93 \mathrm{~Hz}$ was determined which is $20 \%$ lower than the predicted response of $117 \mathrm{~Hz}$. However, a maximum operating frequency of $80 \mathrm{~Hz}(4,800 \mathrm{rpm})$ has been set which is $16 \%$ below the measured first critical. Higher than anticipated bearing clearances account for the lower stiffness and response frequency. Since axial excitation is low during a discharge, no problems were expected.

Radial pocket pressures range from $3,240 \mathrm{kPa}(470 \mathrm{psi})$ on the upper pockets to $5,033 \mathrm{kPa}(730 \mathrm{psi})$ on the lower pockets at an oil temperature of $27^{\circ} \mathrm{C}\left(80^{\circ} \mathrm{F}\right)$. This is expected due to varying clearance caused by the rotor mass. No access to the rotor is available to excite the radial mode with a hammer blow so an alternate means of obtaining a frequency response was employed. The machine was unbolted from the mount, raised $6.4 \mathrm{~mm}(.25 \mathrm{in}$.) above this surface and then quickly lowered to ground. From this test, a $159 \mathrm{~Hz}$ response was obtained for the thrust end radial bearing and $179 \mathrm{~Hz}$ was obtained for the nonthrust end. These values are, respectively, $7 \%$ and $21 \%$ greater than the predicted response of $148 \mathrm{~Hz}$. Therefore, subcritical operation is expected in the radial mode.

\section{Open Circuit Tests}

Prior to performing any rotating machine tests, several static measurements of the armature winding resistance and inductance were taken. Also measured was the transient armature resistance from which the shield resistance may be determined.

To measure the armature winding dc resistance, output from a $40 \mathrm{~V}, 3 \mathrm{~A}$ dc power supply was applied to each winding terminal pair and the voltage drop and current draw for each terminal were recorded. Values between 712 and $724 \mu \Omega$ were obtained for the terminal resistances with an average value of $717 \mu \Omega$. When transposition of the litz wire windings is taken into account, calculations predict a resistance of $674 \mu \Omega$ per terminal which is $6.4 \%$ lower than the measurement. However, of greater importance is the impedance caused by the machine inductance.

Inductance measurements were obtained by capacitor bank discharge through each of the three armature windings. Two tests were performed; one with a $6.3 \mathrm{mF}$ capacitor charged to $180 \mathrm{~V}$ and the other with a $1.05 \mathrm{mF}$ capacitor charged to $250 \mathrm{~V}$. The first test generates a current pulse of $3,400 \mathrm{~A}$ at $168 \mu \mathrm{s}$ with a pulse width of $1.5 \mathrm{~ms}$. The second test resulted in a peak current of $1,500 \mathrm{~A}$ at $86 \mu$ s and a pulse width of $240 \mu s$.

Several tests were performed on each of the three terminal pairs using the capacitor banks described. The values of inductance were obtained by dividing the initial voltage by the rate of change of current and all values were within $10 \%$ of one another. Nominally, the individual winding inductance measures $1.5 \mu \mathrm{H}$. Comparing to the predicted value of $1.2 \mu \mathrm{H}$ results in $25 \%$ difference. However, the higher value could result from the windings of the end turn region being laid edgewise rather than flat as in the active length of the machine.

The machine resistance was also measured using capacitor discharges. For three terminals in parallel, the machine resistance at $4,800 \mathrm{rpm}$ would then be $650 \mu \Omega$. A crude model for the shield resistance assumes image currents in the shield up to a depth equal to the steady state depth of penetration. This model yields an armature winding resistance of $541 \mu \Omega$ which is $20 \%$ lower than our indicated value. More advanced models should be able to predict shield resistance with greater accuracy.

After completing acquisition of static electrical performance data, a series of $500 \mathrm{rpm}$ open circuit motoring tests were performed at field excitation currents ranging from 90 to $1,500 \mathrm{~A}$. Figure 3 shows the open circuit magnetic saturation curve developed from these tests.

Additionally, each terminal voltage was compared and all had the same phase and shape. A peak amplitude variation of $5.0 \mathrm{~V}$ between terminals was detected in a full field test which nominally generated $180 \mathrm{~V}$. While some of this variation can be attributed to differences in instrumentation leads, some voltage differences are expected and will result in circulating currents when connected in parallel to the bus.

Open circuit testing also allowed comparison of actual versus predicted field coil performance. A field current of 401.3 A (O.C. Test $\# 3$, Run \#58) yields a predicted field level of $.691 \mathrm{~T}$. The open circuit voltage is then given by:

$$
\begin{aligned}
& \mathrm{V}_{\mathrm{OC}}=\mathrm{N} 1 \mathrm{v}_{\mathrm{t}} \mathrm{B} \\
& \text { where } \mathrm{N}=\text { number of turns per pole (8) } \\
& \mathrm{N}=\text { number of turns per pole }(8) \\
& v_{t}=\text { rotor tip speed }(21.4 \mathrm{~m} / \mathrm{s} @ 537 \mathrm{rpm}) \\
& \mathrm{B}=\text { field level (0.672--at infinite permeability) } \\
& \text { or } \\
& \mathrm{V}_{\mathrm{OC}}=116.9 \mathrm{~V}
\end{aligned}
$$

This compares well with the actual voltage of $107.8 \mathrm{~V}$ and the difference is due to the fact that the above expression does not account for the actual distribution of the field, but assumes a constant value over the entire armature span. 


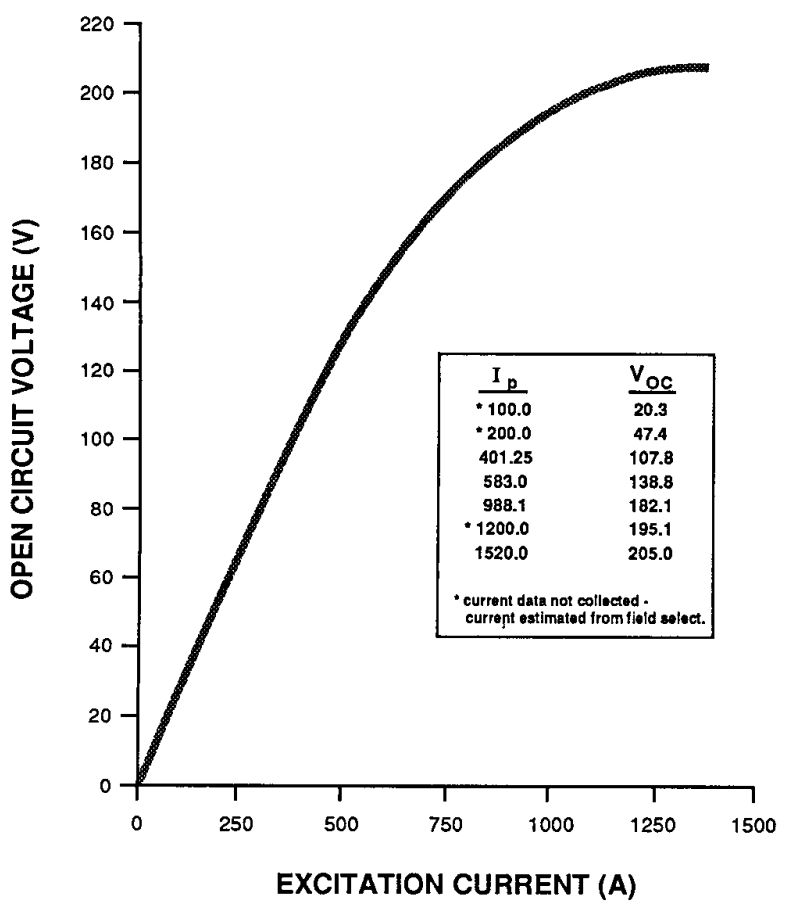

2701.1315 .1

Figure 3. Open circuit magnetic saturation curve for $1 \mathrm{MJ} /$ pulse compulsator (normalized to $495 \mathrm{rpm})$

During O.C. Test \#5 (Run \#62,988 A field), the time constant of the field coil was measured by turning off field current to the coil and continuing to monitor open circuit voltage. The time for the open circuit voltage to decay from $160 \mathrm{~V}$ to $59 \mathrm{~V}(63.2 \%)$ was $\tau=0.88 \mathrm{~ms}$. This compares favorably with a predicted time constant of $1.12 \mathrm{~ms}$.

\section{Discharge Into A Low Impedance Load}

In order to perform a compulsator discharge, an accurate means of triggering the circuit closing switch was required. Imbedded in the stator is an instrumentation coil comprised of a single turn of magnet wire, spanning from one armature pole-center to the next. Two coils were installed so that a backup coil would be available. This coil generates a square-wave $90^{\circ}$ out of phase with the terminal voltage and experiences equal but opposite magnetic flux from the two adjacent armature coils during discharge. A signal conditioning circuit protects against high potential at full machine speed and excitation while allowing operation even at the low voltage induced by the iron residual field. The signal conditioner output is a highly stable TTL-type signal which keys the rotor position.

The accurate determination of the firing angle is achieved by the so named "cycle-portion encoder" developed for this purpose (patent pending). This control is a hybrid analog/digital circuit designed to select a point in a signal cycle based upon a high resolution clock counter rate and the period of the preceding cycle. Given a reliable cyclic input signal, the cycle-portion encoder scales the period of each cycle and steps into the current cycle the desired portion, or firing angle. A detailed description of the stator coil conditioning circuit and cycle portion encoder logic is given in reference 2 .

Figure 4 shows a schematic of the compulsator discharge circuit which, in this case, is an injected electromagnetic launcher. At a given firing angle, the injector switch is closed causing a sliding armature to accelerate in the injector. The firing angle is chosen such that exit of the armature from the injector corresponds to the current crossing at zero. A second current pulse is then initiated (passively--no switching is required) when the armature enters the main railg'n and is completed at armature exit. A typical dis-charge at a rotor speed of $2,400 \mathrm{rpm}$ is shown in figure 5 .

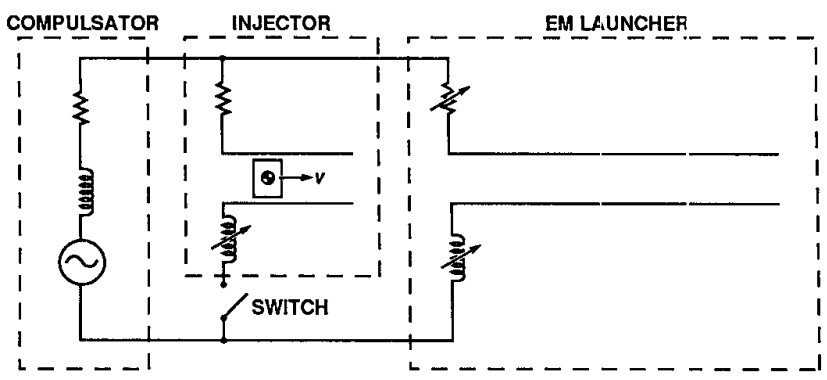

2701.1354

Figure 4. Schematic of the compulsator discharge circuit

UT CENTER FOR ELECTROMECHANICS MACHINE OUTPUT

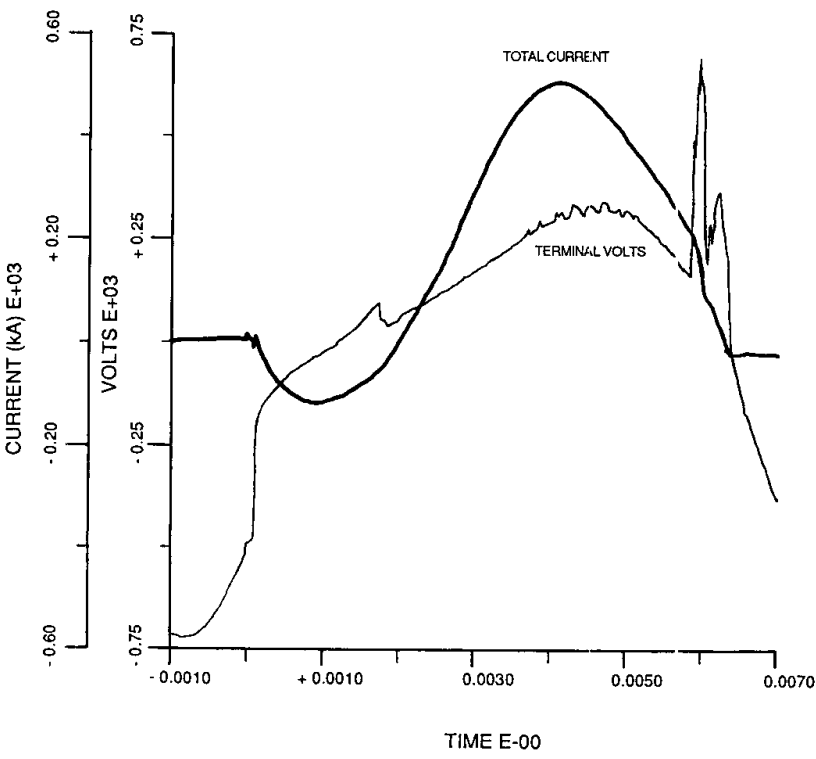

Figure 5. Compulsator discharge into a low impedance inductive load at a rotor speed of 2,400 rprn and $620 \mathrm{~A}$ field current

Confirmation of machine inductance predictions was again attempted by measuring the terminal voltage drop at the time of switch closure and dividing by $\mathrm{di} / \mathrm{dt}$ at this same point. Consistent data from these measurements was not obtained but did indicate that the machine inductance was close to or even lower than estimated. Qualitatively, by observing the expected and actual machine peak currents for a given speed and field, it is apparent that the machine inductance is in line with the predictions. Table 1 illust:ates actual and predicted current levels for various speeds and field levels of the compulsator as well as expected output from an uncompensa ed machine of similar geometry.

At this writing, thirty-three discharges into EN. launchers have been performed by the compulsator at speeds of $2,40 \mathrm{C}, 3,000$, and $3,600 \mathrm{rpm}$ and field currents between 550 and 1,060 A. Since the power and energy level developed by this machine are a function of the speed and field, the most interesting discharge was at 3,600 rpm and 1,060 A field current during Run \#122. 


\begin{tabular}{|c|c|c|c|c|c|c|}
\hline Run & rpm & $\begin{array}{c}\text { Field } \\
(\mathrm{A})\end{array}$ & $\begin{array}{c}\text { Machine Inductance } \\
(\mu \mathrm{H})\end{array}$ & $\begin{array}{c}\text { Predicted Current } \\
(\mathrm{kA})\end{array}$ & $\begin{array}{c}\text { Actual Current } \\
(\mathrm{kA})\end{array}$ & $\begin{array}{c}\text { Uncomp. Machine } \\
\text { Current } \\
(\mathrm{kA})\end{array}$ \\
\hline 176 & 2400 & 710 & 0.745 & 524 & 501 & 82 \\
119 & 3000 & 851 & 0.736 & 634 & 626 & 90 \\
122 & 3600 & 1060 & 0.714 & 759 & 723 & 100 \\
\hline
\end{tabular}

Table 1. Comparison of actual compulsator performance with predicted values including that of a theoretical uncompensated machine of similar geometry

Note: Uncompensated machine current simulation assumes an unsaturated machine inductance of $18 \mu \mathrm{H}$.

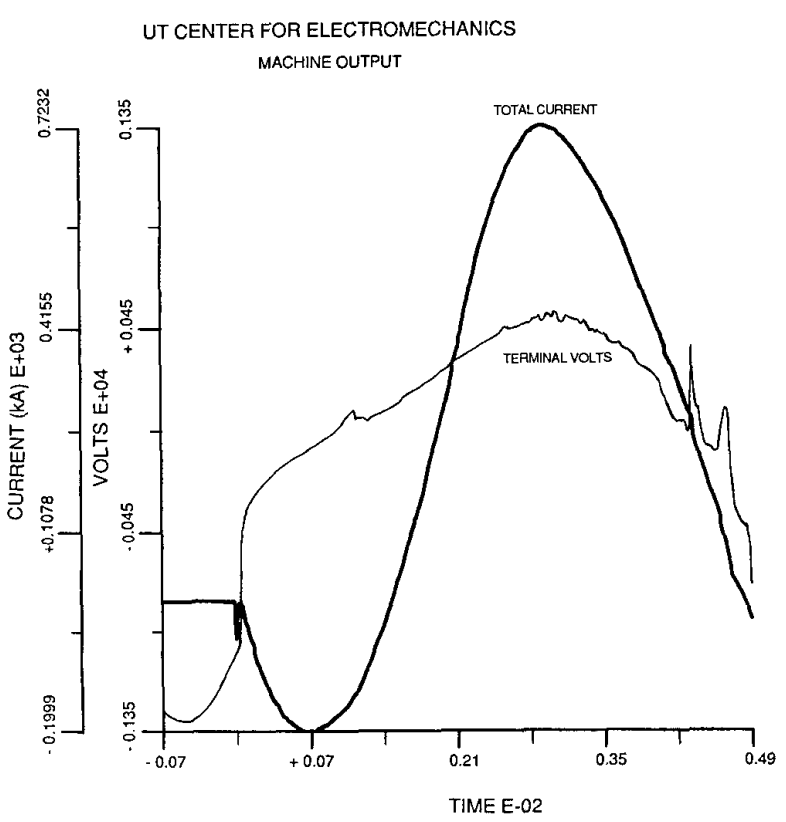

Figure 6. Compulsator Run \#122-curent and voltage performance data

Figure 6 shows the terminal voltage and total current during compulsator Run \#122. Prior to closure of the switch, the open circuit voltage measures $\pm 1,300 \mathrm{~V}$ whereas $\pm 1,360 \mathrm{~V}$ had been predicted. The period of the open circuit voltage just prior to the shot was found to be $5.70 \mathrm{~ms}$, yielding an electrical frequency of $175.4 \mathrm{~Hz}$ or a mechanical speed of $3,510 \mathrm{rpm}$. Accounting for the slower actual speed results in an expected voltage of $\pm 1,323 \mathrm{~V}$ which is less than a $2 \%$ difference from that observed.

Since the driving voltage was less than anticipated, it follows that the current during discharge would be lower than expected. An actual current output of $724 \mathrm{kA}$ was obtained while the simulation predicted $760 \mathrm{kA}$. Reducing the rotor speed in the simulation does reduce the expected peak current but only to $756 \mathrm{kA}$. This is a difference of $4 \%$ from the data.

Multiplying the terminal voltage and total current curves yields the machine power output during the discharge and is shown in figure 7 along with machine current. This shows the peak power delivered to the load was $370 \mathrm{MW}$. Calculating the generated power level is accomplished by multiplying total current times the machine open circuit voltage. This was done by performing a $360^{\circ}$ phase shift of the open circuit voltage data just prior to the shot such that the open circuit voltage and current data were superimposed. Multiplying these two curves results in the theoretical machine power curve shown in figure 8. A peak power of $803 \mathrm{MW}$ was developed by the compulsator of which $46.1 \%$ ( $370 \mathrm{MW}$ ) was transferred to the load.

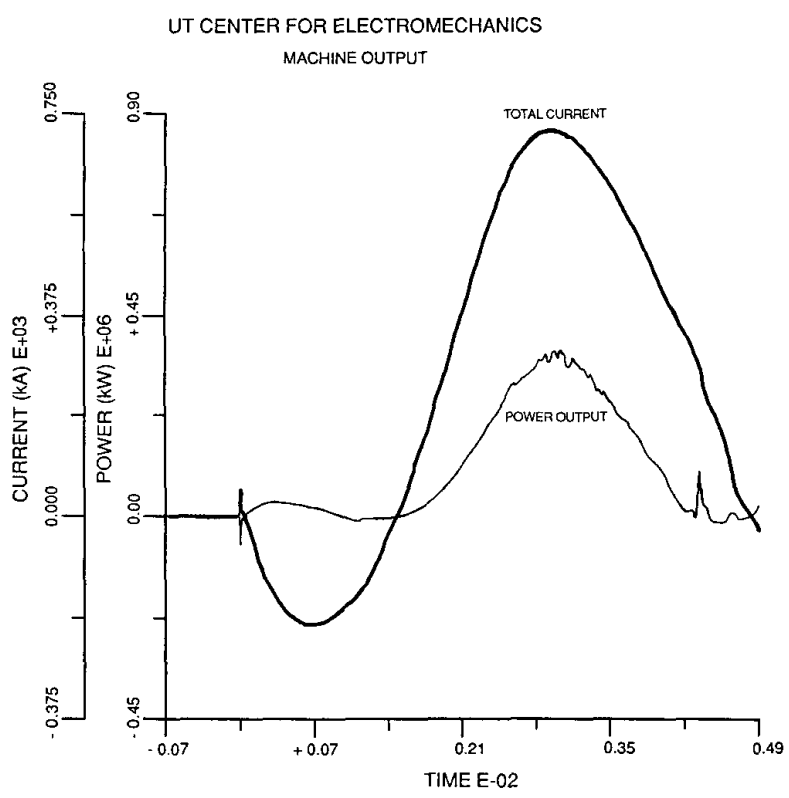

Figure 7. Compulsator run \#122--power output during discharge

Further manipulation of the data yields the total compulsator energy delivered to the load by integrating the actual machine power curve with respect to time. Figure 9 shows the total energy delivered by the compulsator during both the injector pulse and railgun pulse. As can be seen, a peak energy transfer of $1.01 \mathrm{MJ}$ occurs. Note that after each current peak, there is a drop in energy transferred to the load indicating that energy stored in the circuit inductance is being transferred back to the rotor as predicted.

Energy delivery to the load is confirmed by measuring rotor speed before and after the discharge and computing the inertial energy loss of the rotor. Given a rotor inertia of $318 \mathrm{~J}-\mathrm{s}^{2}$ and a change in rotor speed from 3,510 to $3,413 \mathrm{rpm}$ yields a rotor energy loss of $1.17 \mathrm{MJ}$. The difference between this energy and the energy transferred to the load shows a delivered electrical efficiency of $86.3 \%$. 


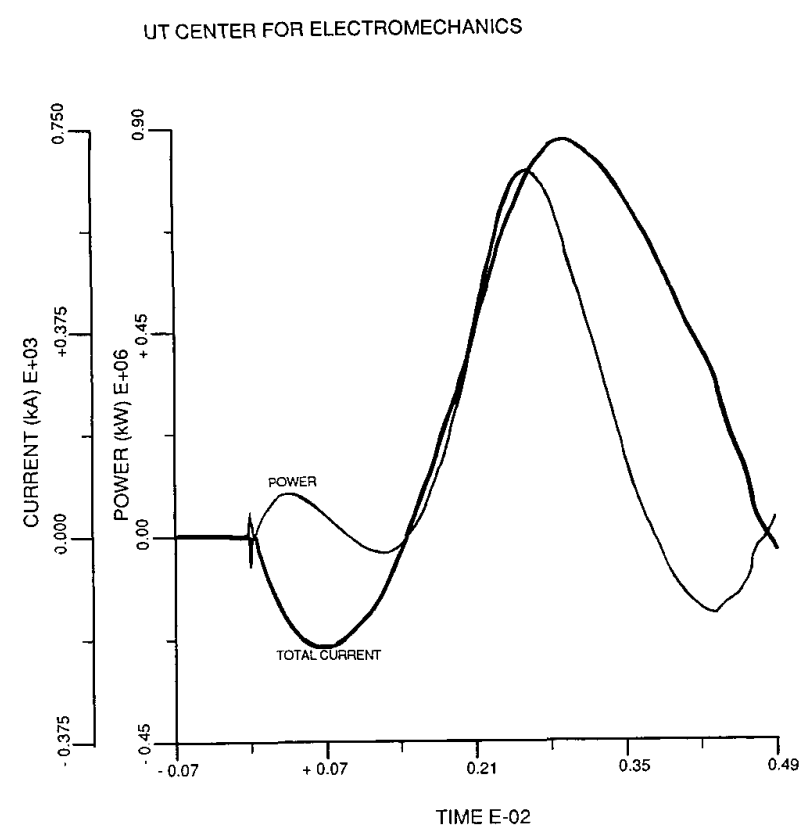

Figure 8. Compulsator run \#122--theoretical power developed by the compulsator

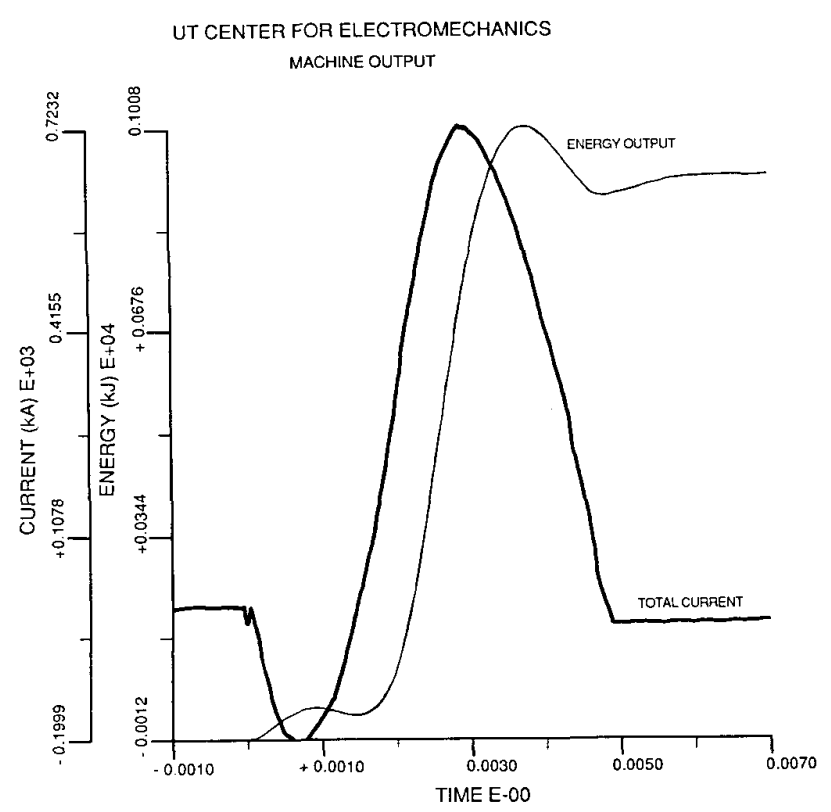

Figure 9. Compulsator run \#122--energy delivered to an inductive load during discharge

As implied by its name, the rapid-fire compulsator is also capable of multiple, high energy discharges at a high repetition rate of $60 \mathrm{~Hz}$. Figure 10 shows the discharge curves of terminal voltage and total current delivered to two EM launchers fired at an interval of 61 $\mathrm{Hz}$. If successive voltage cycles are used to power multiple discharges with the rotor at $4,800 \mathrm{rpm}$, then the compulsator is capable of a $240 \mathrm{~Hz}$ firing rate.

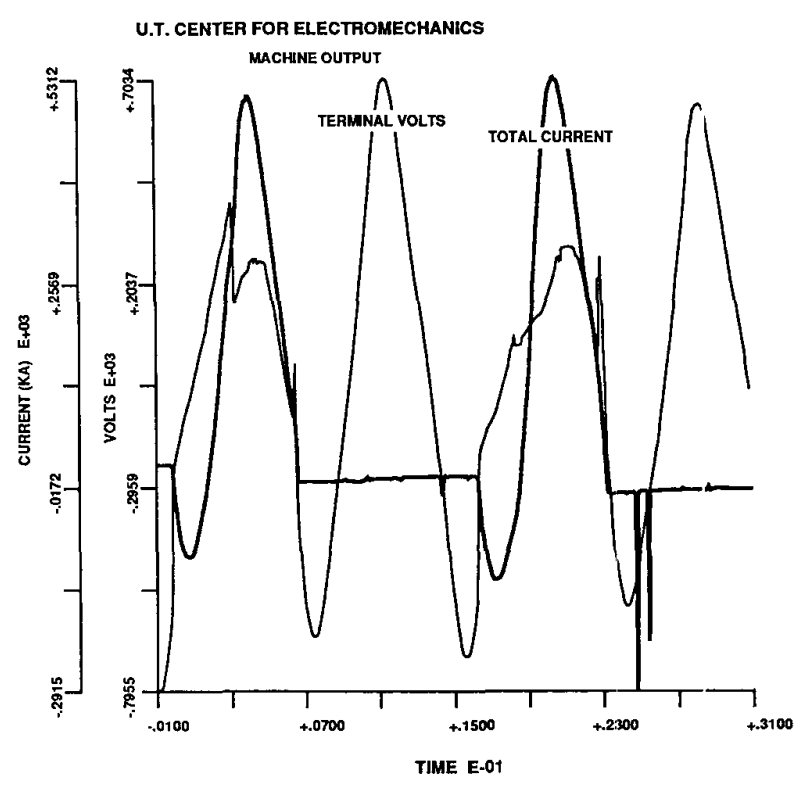

$: 701.1356 .1$

Figure 10. Compulsator Run \#097-current and voltage data for a rapid fire discharge at $61 \mathrm{~Hz}$

For the first shot, the change in rotor speed was frorn 2,488 to $2,413 \mathrm{rpm}$ resulting in a total rotor energy output of $1.28 \mathrm{M}$. For the second shot, the rotor speed went from 2,413 to $2,346 \mathrm{rpm}$ indicating a $1.11 \mathrm{MJ}$ reduction in rotor energy. Discharge energy data derived from Figure 10 shows that 306.7 and $309.7 \mathrm{~kJ}$ were delivered to the two railguns yielding efficiencies of 23.9 and $27.9 \%$. Peak currents of 513 and $532 \mathrm{kA}$ were obtained with the field at $75 \%$ full excitation. Future testing will confirm the ability of the compulsator to deliver multiple, full energy discharges.

\section{Conclusions}

A passively compensated, iron-core compulsator has been built and tested at the Center for Electromechanics at The University of Texas at Austin. This machine has delivered a $1.0 \mathrm{MJ}$ electrical pulse at $724 \mathrm{kA}$ to a low impedance load. At 3,510 rpm, a pulse width of $5.70 \mathrm{~ms}$ is obtained yielding a peak power output of $370 \mathrm{MW}$. Given a machine mass of $11,000 \mathrm{~kg}$, a single discharge into an electromagnetic launcher developed an energy density of $91 \mathrm{~J} / \mathrm{kg}$ and power density of $33.6 \mathrm{~kW} / \mathrm{kg}$. Additionally, multiple discharges at lower encrgy levels have been performed at a repetition rate of $60 \mathrm{~Hz}$.

Trends in compulsator designs are to higher fields and rotating speeds which exceed the mechanical and magnetic constraints of ironbased machines. However, the rapid fire compulsator has proven to be a robust and reliable test bed for high current, high power. electrical loads. Future tests include testing of components such as SCR's for compulsator self-excitation rectifiers as well as continued testing as a power supply for EM launchers and other loads including iasers and particle beam accelerators.

\section{Acknowledgements}

This work was supported by the Defense Advanced Research Projects Agency and the U.S. Army Armament Research, Development and Engineering Center under contract number DAAK10-83-C.0126. The authors also wish to thank Bob Arndt, Jim Long, Brian Rheir, Richard Rodriguez, and Leon Snider for providing invaluable technical assistance throughout the program. 


\section{References}

1. Pratap, S.B., et al., "A Compulsator-Driven Rapid Fire Railgun System," IEEE 5th Pulsed Power Conference, Arlington, VA, June 10-12, 1985.

2. Thelen, R.F., "Pulse Discharge Control and Machine Protection for a Multi-Discharge Compulsator," 4th Symposium on Electromagnetic Launch Technology, Austin, TX, April 12-14, 1988.

3. Bird, W.L., et al., "Applying a Compensated Pulsed Alternator to a Flashlamp Load for Nova-Part II," 2nd IEEE International Pulsed Power Conference, Lubbock, TX, June 12-14, 1979.

4. Carder, B.M., et al., "Driving Parallel Flashlamps with a Compensated Pulsed Alternator," 14th Pulse Power Modulation Symposium, Orlando, FL, June 3-5, 1980.

5. Spann, M.L., et al., "A Rapid Fire, Compulsator-Driven Railgun System," 3rd Symposium on Electromagnetic Launch Technology, Austin, TX, April 20-24, 1986

6. Weldon, W.F., et al., "Compulsator--A High Power Compensated Pulsed Alternator," 2nd International Conference on Energy Storage, Compression, and Switching, Venice, Italy, December 5-8, 1978.

7. Weldon, W.F., et al., "Fundamental Limitations and Design Considerations for Compensated Pulsed Alternators," 2nd IEEE International Puised Power Conference, Lubbock, TX, June 12-14, 1979.

8. Werst, M.D., "Testing of a Rapid Fire Compensated Pulsed Alternator System," 4th Symposium on Electromagnetic Launch Technology, Austin, TX, April 12-14, 1988. 